\title{
CONDITIONS FOR THE COOPER PAIR CREATION IN 2- UP TO 3-DIMENSIONAL FERMI LIQUIDS
}

\author{
R. Gonczarek and M. Mulak \\ Institute of Physics, Technical University of Wroctaw \\ Wybrzeże Wyspiańskiego 27, 50-370 Wrocław, Poland \\ (Received June 14, 1994; in final form September 1, 1994)
}

\begin{abstract}
We reconsider the Pfitzner and Wölfle results concerning the quasi-particle scattering amplitude in Fermi liquid and adopt them to the sufficiently low-temperature and flat-confined systems, where solely two types of quasi-particle interactions are permitted. We find relations between the pairing interaction and Landau parameters and establish plain criteria for the creation of singlet or triplet Cooper pairs. Moreover, we present methods allowing us to estimate values of interaction parameters and transition temperatures in flat-confined systems basing on their bulk parameters. The obtained results are in agreement with experimental data for helium-3 and its films and explain an absence of superconductivity in alkali metals.
\end{abstract}

PACS numbers: 67.50 .Fi

\section{Introduction}

In all investigations of superfluid and superconducting Fermi liquids one always considers two types of quasi-particle interactions, i.e., the Fermi liquid interaction and the pairing interaction. These interactions are treated a priori independently, and parameters of their series expansions in Legendre polynomials are found from experiments for the Fermi liquid interaction (so-called Landau parameters), or postulated with reference to experimental data for the pairing interaction. Such treatment of a problem, though it is very convenient, it is not entirely correct. Pfitzner and Wölfle [1], considering the quasi-particle scattering amplitude from a semi-microscopic model by analytically solving a generalization of Landau's integral equation to momentum transfer up to $2 p_{F}$, showed that there exist general relations between the spin-independent parts of scattering amplitude separated with regard to singlet and triplet components or spin-direct and spin-exchange parts, respectively. However, the mathematical formalism used in such general problem was very involved and complicated, therefore the obtained results could not find a simple application. 
The purpose of this paper is to find the plain relations between the pairing interaction and Landau parameters and explain the problem of cut-off energy, which makes prospects to estimate the superfluid or superconducting properties of a system in virtue of its behaviour in a normal phase. Though the similar efforts were performed in [2-4] the results obtained there were not precise enough to find a direct application. The formalism used herein was improved by Pfitzner and Wölfle [1], therefore the results obtained by us are more consistent and convincing, and they reveal better agreement with experimental data [5]. Moreover, the achieved clarification and simplifications allow us to expand this problem for flat-confined systems and exam the appearing effects connected with reduced dimension [6].

\section{Flat-confined Fermi systems}

In order to consider the above problem in case of flat-confined systems we employ the formalism given in [6], where we established the fractal dimension $d$ where $2 \leq d \leq 3$ for a reverse (momentum) space of such systems and developed analytical methods to research them. In outline, the problem can be presented as follows. We assume that the slab is set parallel to the $x y$-plane and the walls are located at a distance $L$. The separation $L$ can vary from the distance of the order $p_{\mathrm{F}}^{-1}\left(p_{\mathrm{F}}\right.$ is the Fermi momentum in the 3 -dimensional space, cf. [6]) to infinity. It causes that the momentum space is discrete in $p_{z}$ direction and equals $p_{z}=\pi n / L$, where $n$ is an integer [6-9]. The Fermi surface in such space can be constructed as the result of intersection of the discrete space and the Fermi sphere and constitutes the set of concentric circles collocated on the sphere [10-12]. For the specified structure the averaging over the Fermi sphere is of the form [6-9]:

$$
\langle\ldots\rangle_{d}=\int_{0}^{2 \pi} \frac{\mathrm{d} \varphi}{2 \pi} \frac{1}{N_{0}} \sum_{n=0}^{N-1} \ldots,
$$

where $N_{0}=p_{d} L / \pi$ and $N=\left[N_{0}\right]+1\left(\left[N_{0}\right]\right.$ is the nearest integer smaller than $\left.N_{0}\right)$. We also apply the notation $N_{0}=N-\beta$, then $\beta$ increases from 0 to $1(0<\beta \leq 1)$ when $L$ lessens in the suitable range [6]. Moreover, we can define relationship between the dimension $d$ and distance $L$, and for large $L$ we get

$$
d=3-\varepsilon
$$

where $\varepsilon=3 \pi / 4 p_{3} L$ and $\varepsilon \ll 1$.

\section{Connections amongst system parameters}

In any superfluid (superconducting) Fermi systems we can specify three groups of parameters which describe the quasi-particle interactions i.e. Fermi liquid interaction, scattering amplitude and pairing interaction. These interactions after multiplying them by a density of states on the Fermi surface become dimensionless quantities. If we separate their spin components they can be written in the following form which is valid for the confined system, too. 
Fermi liquid interaction:

$\widehat{\boldsymbol{F}}_{d}=F_{d}^{\mathrm{s}}\left(\widehat{\boldsymbol{p}} \cdot \widehat{\boldsymbol{p}}^{\prime}\right) \sigma^{0} \cdot \sigma^{\prime 0}+F_{d}^{\mathrm{a}}\left(\widehat{\boldsymbol{p}} \cdot \widehat{\boldsymbol{p}}^{\prime}\right) \boldsymbol{\sigma} \cdot \boldsymbol{\sigma}^{\prime}$,

Scattering amplitude:

$\widehat{\boldsymbol{R}}_{d}=R_{d}^{\mathrm{s}}\left(\widehat{\boldsymbol{p}} \cdot \widehat{\boldsymbol{p}}^{\prime}\right) \sigma^{0} \cdot{\sigma^{\prime}}^{0}+R_{d}^{\mathrm{a}}\left(\widehat{\boldsymbol{p}} \cdot \widehat{\boldsymbol{p}}^{\prime}\right) \boldsymbol{\sigma} \cdot \boldsymbol{\sigma}^{\prime}$,

Pairing interaction:

$\widehat{\boldsymbol{\Gamma}}_{d}=\Gamma_{d}^{\mathrm{a}}\left(\hat{\boldsymbol{p}} \cdot \hat{\boldsymbol{p}}^{\prime}\right)\left(\mathrm{i} \sigma^{2}\right) \cdot\left({\sigma^{\prime}}^{2} \mathrm{i}\right)+\Gamma_{d}^{\mathrm{s}}\left(\widehat{\boldsymbol{p}} \cdot \hat{\boldsymbol{p}}^{\prime}\right)\left(\mathrm{i} \sigma^{2} \boldsymbol{\sigma}\right) \cdot\left(\boldsymbol{\sigma}^{\prime}{\sigma^{\prime}}^{2} \mathrm{i}\right)$,

where $\sigma^{i}$ are the Pauli matrices ( $\sigma^{0}$ is a unit matrix) and $\widehat{p}, \widehat{p}^{\prime}$ define momentum directions of incoming and outgoing quasi-particles in case of the pairing interaction or two incoming quasi-particles in other cases, respectively. It is well known that the scattering amplitude and Fermi liquid interaction are entirely equivalent. Moreover, we show below that the pairing interaction is not independent of them.

In general case, when the system size is confined and the momentum space is not isotropic, the system can distinguish just one direction $z$ and each scalar function of discussed interactions can be independently expressed as (cf. [6])

$$
X_{d}\left(\hat{\boldsymbol{p}} \cdot \hat{\boldsymbol{p}}^{\prime}\right)=4 \pi \sum_{j=0}^{\infty} \frac{1}{2 j+1} \sum_{m=-j}^{j} X_{j m}(d) Y_{j m}^{*}\left(\theta_{n^{\prime}}^{\prime}, \varphi^{\prime}\right) Y_{j m}\left(\theta_{n}, \varphi\right),
$$

where $Y_{j m}$ are spherical functions, $\theta_{n}=\arccos \left(n / N_{0}\right)$ and $X_{j-m}(d)=X_{j m}(d)$. This expression in the limiting cases $d=3$ or $d=2$ reduces to

$$
X_{3}\left(\widehat{\boldsymbol{p}} \cdot \hat{\boldsymbol{p}}^{\prime}\right)=\sum_{j=0}^{\infty} X_{j} P_{j}\left(\hat{\boldsymbol{p}} \cdot \hat{\boldsymbol{p}}^{\prime}\right)
$$

where $X_{j m}(d)=X_{j}$ for all $m,|m| \leq j$ and $P_{j}(x)$ are the Legendre polynomials, and,

$$
X_{2}\left(\hat{p} \cdot \hat{p}^{\prime}\right)=\chi_{0}+\sum_{m=1}^{\infty} 2 \chi_{m} \cos m\left(\varphi-\varphi^{\prime}\right),
$$

respectively, where $\widehat{p} \cdot \widehat{p}^{\prime}=\cos \left(\varphi-\varphi^{\prime}\right)$ since we put $n=0$. Here

$$
\chi_{m}=\sum_{j=m}^{\infty} \frac{1}{2 j+1} X_{j m}(2) T_{j m},
$$

and (cf. [6])

$$
T_{j m}=\frac{(2 j+1)(j-m) !(j+m) !}{\left[2^{j}\left(\frac{j-m}{2}\right) !\left(\frac{j+m}{2}\right) !\right]^{2}} \frac{1+(-1)^{j-m}}{2} .
$$

In order to standardize parameters of the interactions under consideration we use the following conventional symbols in three-dimensional case. The Fermi liquid interaction parameters, the so-called Landau parameters, have the form

$$
a_{j}^{\mathrm{s}, \mathrm{a}} \equiv \frac{F_{j}^{\mathrm{s}, \mathrm{a}}}{2 j+1}
$$

and satisfy the relation $-1<a_{j}^{\mathrm{s}, \mathrm{a}}<\infty$. The scattering amplitude parameters have the form

$$
r_{j}^{\mathrm{s}, \mathrm{a}} \equiv \frac{R_{j}^{\mathrm{s}, \mathrm{a}}}{2 j+1}
$$


and satisfy the relation $-\infty<r_{j}^{\mathrm{s}, \mathrm{a}}<1$. Moreover, the above parameters are not independent and fulfil the relation [13]

$$
\left(r_{j}^{\mathrm{s}, \mathrm{a}}\right)^{-1}-\left(a_{j}^{\mathrm{s}, \mathrm{a}}\right)^{-1}=1 \text {. }
$$

The pairing interaction parameters have the form

$$
g_{j}^{\mathrm{s}, \mathrm{a}} \equiv-\frac{\Gamma_{j}^{\mathrm{s}, \mathrm{a}}}{2 j+1}
$$

and $g_{j}^{\mathrm{s}, \mathrm{a}} \equiv 0$ for all even, odd $j$, respectively, according to the Pauli exclusion principle. The pairing state (Cooper pairs) can appear only if there exists at least one positive $g_{j}^{\text {s,a }}$ parameter. We do not discard the negative-value pairing parameters $g_{j}^{\mathbf{s}, \mathbf{a}}$ since the existence of them allows us to exclude any coupled states and secondary effects connected with additional coupling constants. Note that the above defined pairing interaction constitutes the generalization of the standard form where it is assumed that the pairing interaction as a function of energy is constant till cut-off energy $\varepsilon_{p}$ and zero above it. However, the pairing interaction defined in such a way contains only positive definite parameters which can be performed in the form $[14,15]$

$$
g_{j}^{\mathrm{s}, \mathrm{a}}=\left(\ln \frac{2 \varepsilon_{p}}{D_{j}^{\mathrm{s}, \mathrm{a}}}\right)^{-1}
$$

where the parameters $D_{j}^{s, a}$ denote values of the pairing energy per quasi-particle in states with the fixed quantum number $j$, therefore $D_{j}^{\mathrm{s}, \mathrm{a}} \equiv 0$ for all even, odd $j$, respectively, and the dominating one can be identified with the ground state energy gap $\Delta$. Though $g_{j}^{\text {s,a }}$ can depend logarithmically on the cut-off energy $\varepsilon_{p}$ which value is not determined univocally for Fermi liquids, and as long as in final results $g_{j}^{\mathrm{s}, \mathrm{a}}$ appear only as independent of $\varepsilon_{p}$, we are fully justified in using them as some physical parameters. On the other hand, the cut-off energy can have physical meaning and e.g. for superconductors it is identified with the Debye energy. Then the above comment is superfluous. Note that the parameters $a_{j}^{\mathrm{s}, \mathrm{a}}, r_{j}^{\mathrm{s}, \mathrm{a}}$ and $g_{j}^{\mathrm{s}, \mathrm{a}}$ tend to zero if $j$ tends to infinity. This statement together with experimental data permits to reduce the number of parameters which are of physical relevance. Let us consider now the relations between the scattering amplitude and pairing interaction parameters in a few most important cases.

\section{Plain relations in three-dimensional case}

Considering a real $d=3$ Fermi liquid system, where the total rotational symmetry is imposed, we should restrict ourselves to sufficiently low temperatures and assume that the energy and momentum of Landau quasi-particles can be taken from the Fermi sphere or its immediate vicinity. It causes that quasi-particle collisions can be realized in two characteristic forms which are equivalent to the physicalscattering amplitude on the Fermi sphere and the pairing interaction, respectively [16]. Since these two types of interactions constitute the limiting cases of the general form of the scattering amplitude considered in [1], we have the right to compare them when they describe identical collisions. This takes place only 
when two quasi-particles with opposite momenta scatter to the same state. However, such approach is only simplification of the problem when we neglect feedback effects between the above interactions (cf. [2-4]). Fortunately, the feedback effects depend on the cut-off energy and they have to disappear for one specific value of $\varepsilon_{p}$ owing to analytical properties of the pairing interaction. Therefore we also have the right to ignore them and fix $\varepsilon_{p}$ if the value of the cut-off energy does not influence final results. Then, after some algebra we get the following relations:

$$
\begin{aligned}
& \sum_{j=0}^{\infty}(-1)^{j}(2 j+1)\left(r_{j}^{\mathrm{s}}+r_{j}^{\mathrm{a}}\right)=-4 \sum_{j=0}^{\infty}(2 j+1) g_{j}^{\mathrm{s}}, \\
& \sum_{j=0}^{\infty}(-1)^{j}(2 j+1)\left(r_{j}^{\mathrm{s}}-3 r_{j}^{\mathrm{a}}\right)=-4 \sum_{j=0}^{\infty}(2 j+1) g_{j}^{\mathrm{a}},
\end{aligned}
$$

which after regarding Eq. (11) allow us to consider $g_{j}^{\text {s,a }}$ as functions of Landau parameters.

Let us verify now the formulas (14) and (15) in relation to helium-3. We assume that solely $g_{0}^{\mathrm{a}}$ and $g_{1}^{\mathrm{s}}$ do not vanish and we take into account just those Landau parameters which are experimentally available in an unshakeable manner, i.e. $a_{0}^{\mathrm{s}}, a_{0}^{\mathrm{a}}$ and $a_{1}^{\mathrm{s}}$. They are functions of pressure and their values are given in Ref. [5]. Though the parameters $g_{0}^{\mathbf{a}}$ and $g_{1}^{\mathbf{s}}$ depend on $\varepsilon_{p}$ they can be estimated numerically according to imposed conditions. However, the obtained results can only be used to identify a sign of the parameters and to observe their dependence on pressure.

In Fig. 1, we present the forms of $g_{1}^{\mathrm{s}}$ and $g_{0}^{\mathrm{a}}$ as functions of pressure. Note that the pairing parameters fulfil the inequalities $0.26<g_{1}^{\mathrm{s}}<0.36$ and $-1.74<g_{1}^{\mathrm{a}}<-1.26$, which prove that there can appear only triplet Cooper pairs.
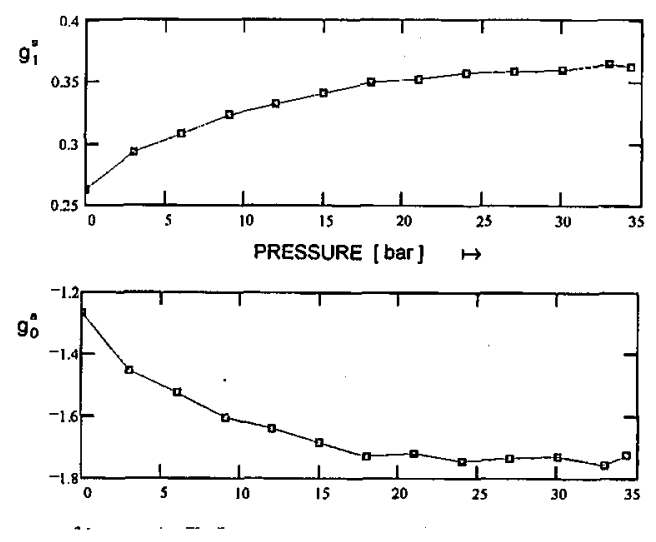

Fig. 1. The presumed forms of the pairing parameters $g_{1}^{s}$ and $g_{0}^{a}$ in helium-3 as functions of pressure for a fixed value of $\varepsilon_{p}$ defined by means of Landau parameters [5]. Note that only $g_{1}^{\mathrm{s}}$ is positive $\left(0.26<g_{1}^{\mathrm{s}}<0.36\right)$ and determines a pairing state whereas $g_{0}^{\mathrm{a}}$ is always negative $\left(-1.26>g_{0}^{\mathrm{a}}>-1.74\right)$ and cannot reveal its existence in any physical effects. 
The most spectacular achievement and advantage of the present formalism is connected with the expression of the critical temperature $T_{\mathrm{c}}$ as a function of pressure by means of the Landau parameters. Taking into account the well-known formula defining transition temperatures in BCS-like systems i.e. $T_{\mathrm{c}}=$ const $\exp \left(-1 / g_{1}^{\mathrm{s}}\right)$, we can transform it to the form

$$
T_{c}(P)=T_{c}\left(P_{0}\right) \exp \left[-\frac{1}{g_{1}^{\mathrm{s}}(P)}+\frac{1}{g_{1}^{\mathrm{s}}\left(P_{0}\right)}\right],
$$

which becomes independent of cut-off energy. $P_{0}$ is an arbitrary value of pressure for which $T_{\mathrm{c}}\left(P_{0}\right)$ has to be taken from experiment. After employing Eq. (14) we find the critical temperature as a function of pressure. The shape of a resulting curve coincides with experimental data and is independent of $\varepsilon_{p}$ which ensures that the used formalism is gauge invariant. Moreover, the curve can be fitted precisely by means of suitable choice of the data, as demonstrated in Fig. 2.

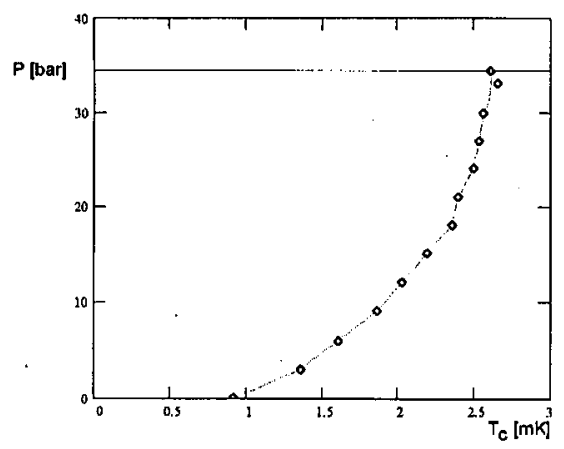

Fig. 2. The phase transition diagram for superfluid helium-3 derived by means of the formula $T_{\mathrm{c}}(P)=T_{\mathrm{c}}(0) \exp \left[-1 / g_{1}^{\mathrm{s}}(P)+1 / g_{1}^{\mathrm{s}}(0)\right]$. For $P=0,3,6, \ldots 33$ and 34.36 bar we have got the following results: $T_{\mathrm{c}}=0.92,1.36,1.61,1.87,2.03,2.20,2.36,2.40,2.49$, $2.53,2.56,2.66$ and $2.61 \mathrm{mK}$ (cf. [5]).

Since the Fermi surface is almost spherical in alkali metals, the Fermi liquid approach can be applied in this case and the Landau parameters can be sensibly defined. However, now the scattering amplitude must be modified in relation to neutral Fermi liquid because of existence of the long-range part of the quasi-particle interaction connected with the Coulomb interaction of electrons. Fortunately, the influence of this interaction, according to [13], can be compensated by putting $r_{0}^{\mathbf{s}} \equiv 1$. Note that though the parameters $g_{j}^{\text {s,a }}$ are probably also modified, they still remain arbitrary and default quantities. In such situation, when applying the developed formalism, we can consider the possibility of creation of superconducting states only with respect to the Landau parameter $a_{1}^{\mathrm{s}}$ and $a_{0}^{\mathrm{a}}$. These parameters are immediately connected with the effective mass (heat capacity) and Fermi liquid spin susceptibility, hence they can be easily evaluated in normal phase. In Fig. 3 we define the regions on $a_{0}^{\mathrm{a}} a_{1}^{\mathrm{s}}$-plane in which the parameters $g_{1}^{\mathrm{s}}$ and $g_{0}^{\mathrm{a}}$ have a fixed sign. Note that there exists a region where the superconductivity (singlet and triplet) cannot be realized at all. The above results allow us to state that 


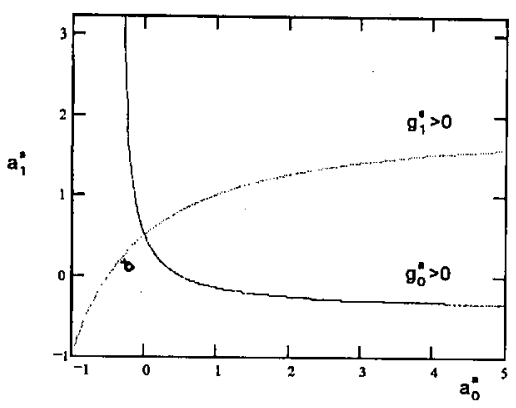

Fig. 3. The regions of a fixed sign of the pairing parameters $g_{0}^{\mathrm{a}}$ and $g_{1}^{\mathrm{s}}$ on $a_{0}^{\mathrm{a}} a_{1}^{\mathrm{s}}$ Landau parameter plane derived for alkali metals. The points $(+)$ and $(\diamond)$ display the values of Landau parameters for $\mathrm{K}$ and $\mathrm{Na}$, respectively [13]. Note that both points lie in the region of negative values of the pairing parameters.

according to data given in Ref. [13] the alkali metals potassium (K) and sodium ( $\mathrm{Na}$ ) can never become superconductors.

\section{Plain relations in two-dimensional case}

In the two-dimensional case (a monolayer system, $L<\pi / p_{d}$ and $d=2$ ) we have to restrict ourselves to one Fermi circle $(N=1$ ), only (cf. [6]). Now, the Fermi liquid interaction, scattering amplitude and pairing interaction can be extended in Fourier series, because of full rotational symmetry in a plane. Hence, the relations (14) and (15) appear now in the form

$$
\begin{aligned}
& \left(\rho_{0}^{\mathrm{s}}+\rho_{0}^{\mathrm{a}}\right)+2 \sum_{j=0}^{\infty}(-1)^{j}\left(\rho_{j}^{\mathrm{s}}+\rho_{j}^{\mathrm{a}}\right)=-8 \sum_{j=0}^{\infty} \gamma_{j}^{\mathrm{s}}, \\
& \left(\rho_{0}^{\mathrm{s}}-3 \rho_{0}^{\mathrm{a}}\right)+2 \sum_{j=0}^{\infty}(-1)^{j}\left(\rho_{j}^{\mathrm{s}}-3 \rho_{j}^{\mathrm{a}}\right)=-4 \gamma_{0}^{\mathrm{a}}-8 \sum_{j=0}^{\infty} \gamma_{j}^{\mathrm{a}},
\end{aligned}
$$

where the coefficients $\rho_{j}^{\mathrm{s}, \mathrm{a}}, \gamma_{j}^{\mathrm{s}, \mathrm{a}}$ and also $\alpha_{j}^{\mathrm{s}, \mathrm{a}}$ are two-dimensional homologues of $r_{j}^{\mathrm{s}, \mathrm{a}}$, $g_{j}^{\mathbf{s}, \mathrm{a}}$ and $a_{j}^{\mathrm{s}, \mathrm{a}}$, respectively, and they are defined according to Eq. (6). Moreover, the Fermi liquid interaction and scattering amplitude parameters fulfil the relation (cf. Eq. (11))

$$
\left(\rho_{j}^{\mathrm{s}, \mathrm{a}}\right)^{-1}-\left(\alpha_{j}^{\mathrm{s}, \mathrm{a}}\right)^{-1}=1,
$$

where $-\infty<\rho_{j}^{\mathrm{s}, \mathrm{a}}<1$ and $-1<\alpha_{j}^{\mathrm{s}, \mathrm{a}}<\infty$. Now, again the pairing interaction parameters $\gamma_{j}^{\mathrm{s}, \mathrm{a}} \equiv 0$ for all even, odd $j$, respectively, according to the Pauli exclusion principle. Therefore, superfluid or superconducting properties of a two-dimensional system can be estimated by means of two-dimensional equivalents of the Landau parameters (cf. Eq. (7)), modifications of which should be determined independently. 


\section{Plain relations in reduced dimension case}

In general case of confined systems $(2<d<3)$ the problem becomes strongly complicated because of a great number of parameters $X_{j m}(d)$ and the lack of information about them. However, if we restrict ourselves to weak-confined systems only, i.e., when $\varepsilon=3 \pi / 4 p_{3} L \ll 1$, we can find relations between parameters of scattering amplitude and Fermi liquid interaction and establish the Pomeranchuk-type stability conditions in standard manner (cf. [6]). The obtained relations have the following form:

$$
\begin{aligned}
r_{j m}^{\mathrm{s}, \mathrm{a}}(3-\varepsilon) & =a_{j m}^{\mathrm{s}, \mathrm{a}}(3-\varepsilon)-a_{j m}^{\mathrm{s}, \mathrm{a}}(3-\varepsilon)\left(1+\frac{2}{3} \varepsilon T_{j m}\right) r_{j m}^{\mathrm{s,a}}(3-\varepsilon), \\
r_{j m}^{\mathrm{s}, \mathrm{a}}(3-\varepsilon) & <1-\frac{2}{3} \varepsilon T_{j m}, \\
a_{j m}^{\mathrm{s}, \mathrm{a}}(3-\varepsilon) & >-\left(1-\frac{2}{3} \varepsilon T_{j m}\right),
\end{aligned}
$$

where the above parameters are defined according to prescription given in Eqs. (9)-(10). Let us represent them in the following form:

$$
\begin{aligned}
& r_{j m}^{\mathrm{s}, \mathrm{a}}(3-\varepsilon)=r_{j}^{\mathrm{s}, \mathrm{a}}\left(1-\varepsilon s_{j m}^{\mathrm{s}, \mathrm{a}}\right), \\
& a_{j m}^{\mathrm{s}, \mathrm{a}}(3-\varepsilon)=a_{j}^{\mathrm{s}, \mathrm{a}}\left(1-\varepsilon b_{j m}^{\mathrm{s}, \mathrm{a}}\right),
\end{aligned}
$$

where $s_{j m}^{\mathrm{s} \text {, a }}$ and $b_{j m}^{\mathrm{s}, \mathrm{a}}$ are defined for $\varepsilon=0$. From Eq. (20) after employing Eq. (11) and Eq. (22) we obtain the following equation:

$$
\frac{s_{j m}^{\mathrm{s}, \mathrm{a}}}{r_{j}^{\mathrm{s,a}}}-\frac{b_{j m}^{\mathrm{s,a}}}{a_{j}^{\mathrm{s}, \mathrm{a}}}=\frac{2}{3} T_{j m}
$$

which allows us to state that the following solution:

$$
s_{j m}^{\mathrm{s}, \mathrm{a}}=b_{j m}^{\mathrm{s}, \mathrm{a}}=\frac{2}{3} T_{j m}
$$

fulfils Eq. (20) for all values of $r_{j}^{\mathrm{s}, \mathrm{a}}$ or $a_{j}^{\mathrm{s}, \mathrm{a}}$ and satisfies the Pomeranchuk-type stability conditions. Note that the above solution is equivalent to the statement that the quasi-particle interaction is proportional to fractal volume of the momentum space.

Considering our results with respect to superfluid helium- 3 we ascertain that the pairing interaction parameter has to be modified according to the same rules as the rest and we get

$$
\begin{aligned}
& g_{11}^{\mathrm{s}}=g_{1-1}^{\mathrm{s}}=g_{1}^{\mathrm{s}}(1-\varepsilon), \\
& g_{10}^{\mathrm{s}}=g_{1}^{\mathrm{s}} .
\end{aligned}
$$

These results imply that the effective value of the pairing interaction decreases in flat-confined systems and hence the estimations of transition temperatures give lower values of $T_{\mathrm{c}}$ in comparison to those obtained in [9] which should allow us to improve the agreement of theory developed in [9] with experimental data [17].

In relation to alkali metals, for which flat-confined systems can be identified with thin metallic films, we conclude that the proportional alterations of all interaction parameters do not affect an equilibrium state. 


\section{Conclusions}

The presented formalism proves that the Fermi liquid and pairing interaction correlate which implies that the Fermi liquid interaction cannot be ignored in superfluid or superconducting systems. Hence, the superfluid state never can be realized in a free quasi-particle gas. Moreover, we showed that the cut-off energy, which manifests as a non-physical parameter in the theory of superfluid helium-3, can be always eliminated from these results for which we can give physical interpretation. Therefore, in accordance with Ref. [15] it never determines behaviour of a system. The formulas presented in our paper constitute the simple medium which permits to predict the appearance of phase transitions to a superfluid or superconducting state in a wide class of 2- up to 3-dimensional Fermi liquid systems and enables to study properties of new states exploiting their static and kinetic quantities defined in a normal phase. We also should mention that within the frame of our approach merely quantitative modifications of a system state induced by its size, such as transition temperature or critical thickness, are available (cf. [8]). Only one aspect of this problem has been for the first time discussed in the present paper and further investigation is needed.

\section{Acknowledgment}

One of us (R.G.) is greatly indebted to Dr G.R. Evans for comprehensive technical help in realization of this work during author's stay in Staffordshire University U.K. in 1994.

This research has been supported in part by grant PB 1444/2/91 and in part by grant 2 P302 12506 of the State Committee for Scientific Research (Republic of Poland).

\section{References}

[1] M. Pfitzner, P. Wölfle, J. Low Temp. Phys. 51, 535 (1983).

[2] B. Patton, A. Zaringhalam, Phys. Lett. A 55, 79 (1975).

[3] K. Levin, O.T. Valls, Phys. Rev. B 32, 105 (1979).

[4] K. Levin, O.T. Valls, Phys. Rev. B 32, 120 (1979).

[5] J.C. Wheatley, Rev. Mod. Phys. 85, 415 (1975).

[6] R. Gonczarek, I.H. Krzyżanowski, Phys. Lett. A 171, 107 (1992).

[7] R. Gonczarek, Physica B 169, 527 (1991).

[8] R. Gonczarek, I.H. Krzyżanowski, Acta Phys. Pol. A 80, 553 (1991).

[9] R. Gonczarek, I.H. Krzyżanowski, Phys. Status Solidi B 165, K5 (1991).

[10] Z. Tes̆anović, Phys. Rev. B 32, 7575 (1985).

[11] Z. Tešanović, O.T. Valls, Phys. Rev. B 33, 3139 (1986).

[12] Z. Tešanović, O.T. Valls, Phys. Rev. B 34, 7610 (1986).

[13] P.M. Platzman, P.A. Wolff, Wave and Interaction in Solid State Plasmas, Academic Press, New York 1973, Ch. 11. 
[14] J. Czerwonko, Acta Phys. Pol. 32, 335 (1967).

[15] D. Rainer, J. Serene, Phys. Rev. B 13, 4745 (1976).

[16] R. Gonczarek, Phys. Status Solidi B 139, K15 (1987).

[17] C.S. Steel, P. Zawadzki, J.P. Harrison, A. Sachrajda, Physica B 165/166, 599 (1990). 